is heterogeneous, the coefficient of absorption in aluminium of the softest and hardest components being respectively $39.7 \mathrm{~cm}^{-1}$ and $0.051 \mathrm{~cm}^{-1}$. In order to determine the nature of the radiation scattered from air it is being subjected to a magnetic field, and experiments of this kind are in progress.

Some experiments to determine the effect upon ionisation measurements of lining the electroscope with different substances have also been made. A comparison of the curves, showing the transmission of radiation through lead obtained with an electroscope $(a)$ unlined and $(b)$ lined with cardboard 0.18 $\mathrm{cm}$. thick, affords very definite information about the quality of the radiation responsible for the ionisation observed. It was found that, when the ionisation was due to radiation of the same wave-length as the primary gamma rays, the insertion of the lining increased the apparent transmission of a filter, the transmission value for a lead filter $10 \cdot 1 \mathrm{~cm}$. thick being 1.27 times that found with the lining removed. When the ionisation was due partly to scattered radiation, the apparent transmission of the filter was decreased by inserting the lining into the electro. scope. The unlined electroscope in this case gave a transmission figure for a lead filter $6.0 \mathrm{~cm}$. thick, which was 1.39 times that found when the electroscope was lined with cardboard. One has therefore a means, likely to prove useful in absorption experiments, of detecting any change in the quality of the radiation entering the electroscope, with the thickness of the filter placed in the primary beam.

Physics Department,

The Middlesex Hospital,

London, W.1.

\section{Multiple Chromosomes and Reduction Division in Flowering Plants.}

IT has been the general practice to speak of the chromosomes shown in the metaphase plates in the root-tips (and other somatic tissues) of flowering plants as single chromosomes. They are never, it spems, called bivalents, dyads, or disomes; though they always show a fine longitudinal split distinctly, in correctly fixed preparations. The chromosomes of the late prophase and first metaphase of the maturation divisions in flowering plants have, in my opinion, a close parallelism to those of the somatic divisions; in that the two chromatids of any one of the two homologous chromosomes are rendered visible only through the appearance of a fine longitudinal split, seen in correctly fixed preparations.

Hence it seems we need not necessarily call such a split chromosome (in flowering plants) a bivalent, dyad, or disome. It seems more convenient to call it a single chromosome, as in the case of the somatic division, and to speak of its two halves as chromatids, not as chromosomes; and this in fact seems to be the usual practice. Similarly, at the first anaphase and second metaphase, where the two chromatids have usually separated, except at the points of constriction, we usually, it seems, call the double $V$, etc., as it proceeds to the pole, a single chromosome. When it appears again in the second metaphase plate, as a double $V$, or a four-lobed cross, we again usually call it a single split chromosome. Hence we may perhaps define a single individual chromosome as normally consisting of two halves (chromatids) which have not yet completely separated. When they have completely separated, we have two chromosomes.

The reverse does not hold. For when two completely separate chromosomes come together (as in the prophase of maturation) they do not form a single chromosome, but a bivalent, dyad, or disome; and show double in well-fixed preparations. (The above seems to embody the prevailing usage with regard to flowering plants.)

The reduction division, in one of the old senses of the term-namely, reduction in the number of each gene from two to one-has, with the discovery of segmental interchange (crossing-over), ceased to have a signification, as Bridges has already pointed out.

In the second meaning of the term, namely, a reduction in the number of chromosomes, it seems still available. Using the limits of the term 'chromosome' as defined above, at the first maturation division the reduction is from nuclei with $n$ bivalents, which must of course count as $2 n$ chromosomes, to nuclei containing only $n$ chromosomes. Hence the reduction division in the diploid flowering plants yet examined is the first of the two maturation divisions. This is confirmed by true triploid plants, in which the reduction metaphase has $n$ trivalents, giving $3 n$ chromosomes, as defined above. There are formed, after reduction, nuclei with groups of split chromosomes, each consisting of $2 n-p$, where $p$ has the values from 0 to $n$. Here again there is a reduction from $3 n$ to something between $2 n$ and $n$. Also in some haploid plants at least, with $n$ single chromosomes (each presumably consisting of two chromatids), these chromosomes proceed to either pole without distinctly splitting, and split there. In this case there is a reduction from $n$ to $n-p$, where $p$ has the values from 0 to $n$.

JoHN Belline.

Carnegie Institution of Washington,

Department of Genetics,

Cold Spring Harbour, N.Y.

\section{Colorimetric Determination of the Oxidation of Hæmocyanin.}

IN a recent paper confirming a previous finding by myself (British Journal of Experimental Biology, vol. 3,1925$)$ to the effect that the hæmocyanin of Cancer has minimum affinity for oxygen at a $p \mathrm{H}$ circa 7.0, Stedman and Stedman (Biochemical Journal, vol. 20, 1926) make the following comment on the colorimetric method:

"Although the colorimetric method might be expected to give accurate results and possesses the advantage of being rapid and simple, it is evident that curves obtained by this method under different conditions of acidity will not be comparable unless the standards are in each case maintained at the same degree of saturation. This condition will not be fulfilled in the case of hæmocyanin from Cancer and Homarus, if the standards are in equilibrium with air and the temperature is as high as $23^{\circ} \mathrm{C}$. It is improbable that they were fulfilled at the slightly lower temperature of $18.7^{\circ}$ employed by Hogben in the case of Cancer serum. . . . While the colorimetric method is undoubtedly capable of indicating the general influence of $p H$ on a particular hæmocyanin, the results so obtained can have no quantitative significance unless care is taken to avoid the sources of error indicated above."

This criticism is surprising in view of the fact that the standards used, as explicitly mentioned both in published statement and in personal communication to the Stedmans, were prepared from normal serum of Cancer. It has been shown by these authors (Biochemical Journal, vol. 19, p. 547) that the normal serum of Cancer at room temperature is 88.1 per cent. saturated at an oxygen partial pressure of $21.7 \mathrm{~mm}$., and 100 per cent. saturated at $80 \mathrm{~mm}$., i.e. at half the partial pressure of oxygen in the atmosphere. 